\title{
On the Anticipatory Aspects of the Four Interactions: what the Known Classical and Semi-Classical Solutions Teach us.
}

\author{
Luca Lusanna \\ Sezione INFN di Firenze \\ L.go E.Fermi 2 (Arcetri) \\ 50125 Firenze, Italy \\ E-mail: LUSANNA@FI.INFN.IT
}

\begin{abstract}
The four (electro-magnetic, weak, strong and gravitational) interactions are described by singular Lagrangians and by Dirac-Bergmann theory of Hamiltonian constraints. As a consequence a subset of the original configuration variables are gauge variables, not determined by the equations of motion. Only at the Hamiltonian level it is possible to separate the gauge variables from the deterministic physical degrees of freedom, the Dirac observables, and to formulate a well posed Cauchy problem for them both in special and general relativity. Then the requirement of causality dictates the choice of retarded solutions at the classical level. However both the problems of the classical theory of the electron, leading to the choice of $\frac{1}{2}$ (retarded + advanced) solutions, and the regularization of quantum field teory, leading to the Feynman propagator, introduce anticipatory aspects. The determination of the relativistic Darwin potential as a semi-classical approximation to the Lienard-Wiechert solution for particles with Grassmann-valued electric charges, regularizing the Coulomb self-energies, shows that these anticipatory effects live beyond the semi-classical approximation (tree level) under the form of radiative corrections, at least for the electro-magnetic interaction.
\end{abstract}

Talk and "best contribution" at The Sixth International Conference on Computing Anticipatory Systems CASYS'03, Liege August 11-16, 2003 


\section{INTRODUCTION}

Our understanding of the four interactions (gravitational, electro-magnetic, weak and strong, with or without super-symmetry) has led to a description of physics which utilizes classical action principles whose associated Lagrangian densities are singular. Therefore the equations of motion for the fields and/or the particles cannot be written in normal form, namely they cannot be solved for the accelerations and put in the form of the equations of Newtonian mechanics $(m \vec{a}=\vec{F})$. This is due to the requirements of manifest Lorentz covariance and gauge invariance for special relativistic systems and of general covariance for general relativistic systems. To comply with them we have to introduce redundant nonphysical quantities which transform covariantly like tensors under the appropriate group. In special relativistic particle theory we must use gauge potentials $A_{\mu}(x)$ defined modulo gauge transformations and particle world-line coordinates (events) $x_{i}^{\mu}(\tau), i=1, . ., N$, defined modulo reparametrizations of the scalar affine parameter $\tau$. While in the first case two components of the gauge potential are non-measurable mathematical quantities, in the second case we have as many time coordinates $x_{i}^{o}(\tau)$ as particles and we must connect them to the time of the special relativistic clocks in such a way that physical quantities (for instance bound states of the particles) are independent from the unphysical relative times $x_{i}^{o}(\tau)-x_{j}^{o}(\tau)$. The description of bound states must be independent from the freedom of the observer of looking at the particles at the same time or with any prescribed time delay among them [1]. With strong and electro-weak interactions we must use the non-Abelian Lie algebra-valued gauge potentials $A_{a \mu}(x) T^{a}$, with $T^{a}$ a matrix representation of either $S U(3)$ or $S U(2) \times U(1)$, and again for each value of $a$ two components of the gauge potential are gauge variables [2].

Einstein's general relativity and all the existing variants emphasize that, contrary to special relativity where Minkowski space-time is absolute, space-time points loose their physical individuality (the Hole Argument [3] ), that coordinates are purely conventional and that measurable physical quantities must not depend on their choice. These are consequences of the invariance of the theory under active diffeomorphisms and general coordinate transformations (passive diffeomorphisms) and this implies the general covariance of the equations of motion (they must take the same functional form in every coordinate system). This leads to the fact that only two of the ten components of the metric tensor are dynamically deter- 
mined by Einstein's equations in every coordinate system. As a consequence there is a going on debate on which are the observables in general relativity (see for instance Ref.[4]), in particular for the gravitational field itself, and on how to build a reference standard (Global Positioning System?) for an empirical determination of a system of coordinates with the associated metric tensor to be used for every subsequent measurement of times, lengths, angles, tidal and inertial effects of the gravitational field, matter properties. In Refs. [5] there is a complete discussion of these problems and a proposal for their solution based on a formulation of metric 6] and tetrad [7, 8] gravity in globally hyperbolic, topologically trivial, asymptotically flat at spatial infinity space-times. It is shown that in these space-times, be-

longing to the family of Christodoulou - Klainermann space-times [9], it is possible to define a rest-frame instant form of gravity, where the evolution is governed by the ADM energy (they are a counter-example to the frozen picture without evolution of general relativity).

\section{GAUGE VARIABLES AND DIRAC OBSERVABLES}

The main consequence of this state of affairs is that both in special and general relativity the covariant equations of motion do not determine the unphysical gauge degrees of freedom present in the covariant description of the system. This fact gives rise to a lack of determinism in the time evolution of the covariant variables. As a consequence, one has to face the problem to disentangle the arbitrary gauge variables from the deterministic physical measurable quantities (Dirac observables), whose equations of motion in the form of a hyperbolic system of (partial) differential equations with a well posed Cauchy problem on a space-like initial hyper-surface of space-time. Once this is done, the next problem is the empirical impossibility of knowing (i.e. preparing) all the physical initial data on a noncompact Cauchy surface: this the price to be paid to get the theorems on the existence and uniqueness of the solutions of the equations of motion.

The natural environment where to formulate, study and try to solve these problems at the relativistic level is not configuration space but phase space. Here the theory of singular Lagrangians, due to the second Noether theorem[10], gives rise to Dirac-Bergmann theory of Hamiltonian constraints [1] by means of which a well defined formulation of the lack of determinism of every special or general relativistic theory can be given in the framework of Hamilton-Dirac equations of motion. Moreover the family of Shanmugadhasan canonical 
transformations [12] allows, at least at a heuristic level in field theory, to find special canonical bases in which: a) a subset of the new momenta are the generators of the Hamiltonian gauge transformations (Abelianized form of the first class constraints); b) the conjugated configurational variables are an Abelianized form of the redundant gauge variables; c) the remaining pairs of canonical variables form a Darboux basis of Dirac observables (no method is known in configuration space to find a basis of observables). The Dirac observables are the physical measurable quantities which have a deterministic evolution, since they satisfy hyperbolic Hamilton equations with a completely determined Hamiltonian and with a well posed Cauchy problem. However, in general, they are not covariant, namely their functional form is gauge dependent. In special relativity it is known how to find covariant functions of the Dirac observables (for instance in electromagnetism such functions are the transverse electric and magnetic fields). Instead in general relativity it is still an open problem how to build functions of the Dirac observables of the gravitational field which do not depend on the choice of the coordinates. In the first of Refs. [5] there is the conjecture that in gravity there are special Shanmugadhasan canonical bases, in which both the Dirac observables and the gauge variables (describing generalized tidal and inertial effects respectively) are coordinate-independent: they should emerge from a Hamiltonian re-formulation of the Newman-Penrose approach [13].

\section{THE INITIAL VALUE PROBLEM AND ANTICIPATION}

Modulo manifest covariance Dirac observables are the deterministic relativistic counterpart of the configurational or phase space variables of ordinary Newton mechanics, both classically and quantum mechanically. Therefore, it is only at the level of Dirac observables

that the problematic about anticipation is well posed (see the review paper of Dubois [14] on anticipatory systems). Moreover, numerical simulations and/or approximations with finite difference equations, with their possible change of perspective on the properties of solutions, are physically motivated only for the Hamilton equation for Dirac observables.

Given the deterministic equations of motion of an isolated system we have first of all to choose definite boundary conditions so that the functional space in which to look for solutions is well defined (this step usually is trivial in mechanical problems). Then we have to formulate a well posed initial value problem. The two main classes of such problems 
are connected with the names of Cauchy and Dirichlet. In a Cauchy problem for a second order (partial) differential equation we have to give the initial configuration and the initial velocities on the space-like hyper-surface corresponding to the initial time (in relativistic theories this implies a choice of equal time (simultaneity) Cauchy surface and the description of the relativistic system is acceptable if the physical results do not depend on this choice). Then causality induces the choice of retarded Green functions in the construction of solutions to the given problem, which therefore do not depend on events in the absolute future of the initial configuration of the system. However, as noted in Ref.[14], for simple mechanical systems the equations of motion may depend on parameters, which a posteriori can be seen to coincide with the value of some configurational variable at a later final time. In these cases, some type of anticipation is present in the system.

Instead in a Dirichlet problem we look for solutions of the equations of motion determined by an initial configuration of the system at time $t_{i}$ and by a final one at time $t_{f}>t_{i}$. Clearly in this type of solutions we have anticipation, since the knowledge of the future configuration determines the intermediate configurations. The formulation of Dirichlet problems is a much harder task than the Cauchy problem, since all the topological properties of the intermediate configurations have to be known in advance.

Classical relativistic theories, relying on the standard notion of causality, tried to avoid anticipation by using only retarded solutions of the classical relativistic wave equations (in them there are no parameters hiding anticipation). However this attempt was frustrated by the problems of the classical theory of the electron. The Abraham-Lorentz-Dirac equation, giving the correct Larmor formula for the radiation in wave zone, depends on the time derivative of the acceleration. To avoid runaway solutions, with their unlimited growing of the acceleration, we have to re-formulate the equation as an integral equation, and this creates a problem with causality (the pre-acceleration, see for instance Ref.[15]), which is usually solved by refuting the validity of the classical theory and invoking quantum mechanics. Moreover, in the limit of a point-like electron we have the appearance of the infinities connected with the Coulomb self-energies.

These problems of the classical theory were debated for a century and the only way out was Feynman-Wheeler electrodynamics [16] with their theory of the absorbers. Here anticipation is present, because the chosen solutions admit a symmetric combination of retarded and advanced effects. 
The whole problematic was re-formulated with quantum field theory regularized in such a way to eliminate the infinities. This is a theory with anticipation, because, due to the fact that no one has been yet able to regularize the product of two retarded distributions, it uses Feynman Green functions, whose real part is the symmetric combination of retarded and advanced Green functions (the imaginary part is connected to diffractive absorption in the other channels of the many-body theory). The same happens in the formulation of the theory by means of path integrals, since they correspond to a Dirichlet problem, in which the initial and final configurations are given. Let us remark that, notwithstanding all the successes of quantum field theory, we do not yet know whether the causal problems of the classical theory are solved or not. In perturbative quantum electrodynamics they are absent by construction. But the perturbative expansion is only an asymptotic series which cannot be resummed. On the other hand at the non-perturbative level (the path integral approach) we do not know how to formulate the problem.

Moreover quantum field theory is a special relativistic theory and it is not known how to incorporate gravity in it in a regularized way which takes into account the already quoted problems of the physical identification of space-time points. See the first of Refs. [5] for a proposal of quantization of gravity preserving relativistic causality.

Indeed our understanding of quantum theory requires that the events (the points) of space-time and the simultaneity Cauchy surface be fixed in advance in an absolute way. For instance there are suggestions (see Penrose contribution in Ref.[17]) that gravity may play a role in understanding the unsolved problem of how the potentialities of quantum theory (which gives only probabilities) become the actualities we experience (the problem of the collapse of the wave function when we do a measurement on an entangled state, consequence of the linearity of the Scrhoedinger equation and of the superposition principle). Another unsolved problem is the integrability of 4-dimensional classical and quantum field theories and the repercussions of chaotic motions on both the regularizzability of quantum field theories and on our understanding of gravitational physics.

Therefore our present knowledge about physical systems points in the direction of the presence of anticipation, but also stimulates us to understand better the interface between classical and quantum systems, especially at the relativistic level in view of the inclusion of gravity. 


\section{THE REST-FRAME INSTANT FORM OF DYNAMCS}

Therefore I started a research program aiming to arrive at a unified description of the four interactions at the classical level in terms only of Dirac observables (see Ref.[2] for a review). The first stage was to re-formulate all classical isolated systems (particles, strings, fields) on arbitrary space-like hyper-surfaces (corresponding to a concept of simultaneity for a family of time-like non-inertial accelerated observers) instead that on space-like hyperplanes (inertial observers). This was done in such a way that physical results are independent from the choice of the hyper-surface (special relativistic general covariance of parametrized Minkowski theories). In this way it is possible to add the gravitational field to the description in such a way that the switching off of Newton constant allows to recover the isolated system without gravity (solution of the deparametrization problem of general relativity).

Then I have studied the description of the isolated systems in special relativity when they are restricted to the special family of space-like hyper-planes orthogonal to the conserved 4-momentum of the system. These so-called Wigner hyper-planes are determined by the isolated system itself and define its intrinsic rest frame [18]. They opened a new viewpoint to understand the old unsolved problem of which is the best definition of the relativistic center of mass and allowed the identification of new kinematical variables both for the Nbody problem and for extended systems [19]. Moreover in this way it was possible to define a new instant form of dynamics in Dirac sense [20], the Wigner covariant 1-time rest-frame instant form [2, 18] and to identify an intrinsic classical unit of length for extended rotating systems, the Møller radius [21], which hopefully will be used as a physical ultraviolet cutoff to regularize infinities [2]. At the classical level the Møller radius identifies a world-tube which contains the region of non-covariance of the relativistic center of mass (the classical counterpart of the Newton-Wigner position operator) and it can be shown to be the remnant in flat space-time of the energy conditions of general relativity. At the quantum level it is proportional to the Compton wavelength of the system, so that it impossible to localize the non-covariant center of mass without producing quantum pairs. Therefore, the world-tube, which is a consequence of the existence of the light cone (Lorentz signature of space-time, constancy of the velocity of light), lies at the intersection between quantum theory and general relativity.

The next step of the program was the determination of the Dirac observables of the var- 
ious theories 2]. This has been accomplished for the standard $\mathrm{SU}(3) \mathrm{xSU}(2) \mathrm{xU}(1)$ model of elementary particles (electro-magnetic, weak and strong interactions) in special relativity on the Wigner hyper-planes. Now, after the definition of the rest-frame instant form of metric 6] and tetrad [7, 8] gravity, the Dirac observables for the gravitational field are under investigation and we begin to understand how to identify the points of space-time a posteriori by using the gravitational field. A first byproduct has been the definition of backgroundindependent gravitational waves in post-Minkowskian space-times [22] by means of a Hamiltonian linearization of tetrad gravity in a completely fixed non-harmonic 3-orthogonal gauge. The final step will be to put together the standard model of elementary particles and the gravitational field. Then one will face the problem of quantization.

\section{A SEMI-CLASSICAL DESCRIPTION OF CHARGED PARTICLES PLUS THE ELECTROMAGNETIC FIELD}

In the meanwhile I revisited the classical problem of charged particles plus the electromagnetic field, which was the source of anticipation in physics, in the rest-frame instant form on Wigner hyperplanes [23, 24]. To avoid infinities in the classical self-energies, in place of an extended electron model a semi-classical description of the electric charge with Grassmann variables was used [25]. Experimentally the electric charge is quantized: if magnetic monopoles exist the product of the electric and magnetic charges is proportional to the Planck constant; if they do not exist, we do not understand the quantum of electric charge. If we consider a quantum operator (like the spin or the electric charge) whose spectrum has a finite number of discrete levels, we cannot recover the classical theory by going to high quantum numbers. However, we can get a semi-classical description by replacing the operator with a suitable Grassmann variable (Grassmann variables $Q_{i}$ are mathematical objects satisfying $Q_{i} Q_{j}+Q_{j} Q_{i}=0$ so that $\left.Q_{i}^{2}=0\right)$ : in this way we get a consistent treatment in which the quantum operator $\hat{Q}$ is replaced by an infinitesimal quantity $Q$ (an infinitesimal quantum) and infinitesimals of higher order are discarded $\left(Q^{2}=0\right)[26]$. With an appropriate quantization rule we can recover the quantum theory with the operator $\hat{Q}$. On the other hand, by means of Berezin-Marinov Grassmann-valued density matrix [27] we can get the classical theory: for the spin of a particle there is no classical analogue, while it exists for the electric charge [25]. 
Therefore we studied the system of $\mathrm{N}$ positive energy particles with Grassmann-valued electric charges $Q_{i}\left(Q_{i}^{2}=0, Q_{i} Q_{j}=Q_{j} Q_{i} \quad 10\right.$ for $\left.i \neq j\right)$ coupled to a dynamical (not external) electro-magnetic field, both in the case of spinless particles [23] and of spinning ones [24] with Grassmann-valued spins [28], in the rest-frame instant form. By means of a Shanmugadhasan canonical transformation the system was expressed only in terms of Dirac observables both for the particles $\left(\vec{\eta}_{i}(\tau), \vec{\kappa}_{i}\right.$ with $x_{i}^{\mu}(\tau)=z^{\mu}\left(\tau, \vec{\eta}_{i}(\tau)\right)$, where the functions $z^{\mu}(\tau, \vec{\sigma})$ describe the embedding of the Wigner hyper-planes in Minkowski space-time) and for the electro-magnetic field (it corresponds to the radiation gauge with transverse vector potential $\vec{A}_{\perp}(\tau, \vec{\sigma})$ and electric fields $\left.\vec{E}_{\perp}(\tau, \vec{\sigma})\right)$.

A first consequence of the Grassmann-valued charges $\left(Q_{i}^{2}=0, Q_{i} Q_{j}=Q_{j} Q_{i} \neq 0\right)$ is the regularization of the Coulomb self-energies (the $i \neq j$ rule) in the rest-frame Hamiltonian. This Hamiltonian is the rest-frame invariant mass of the isolated system. In the case of positive energy spinning particles it was necessary to make a semi-classical Foldy-Wouthuysen transformation to determine how the spin of the positive energy particles couples to the electric field. The form of the Hamiltonian in the two cases is $\left[\vec{\xi}_{i}\right.$ are the Grassmann variables for the description of the semi-classical spin $\left.S_{i}^{r}=-\frac{i}{2} \epsilon^{r u v} \xi_{i}^{u} \xi_{i}^{v}\right]$

$$
\begin{aligned}
& H_{\text {spinless }}=\sum_{i=1}^{N} \sqrt{m_{i}^{2}+\left(\check{\vec{\kappa}}_{i}(\tau)-Q_{i} \check{\vec{A}}_{\perp}\left(\tau, \vec{\eta}_{i}(\tau)\right)\right)^{2}}+ \\
& +\sum_{i \neq j} \frac{Q_{i} Q_{j}}{4 \pi\left|\vec{\eta}_{i}(\tau)-\vec{\eta}_{j}(\tau)\right|}+\int d^{3} \sigma \frac{1}{2}\left[\check{\vec{E}}_{\perp}^{2}+\check{\vec{B}}^{2}\right](\tau, \vec{\sigma}), \\
& H_{\text {spin }}=\sum_{i=1}^{N}\left[\sqrt{m_{i}^{2}+\left(\check{\vec{\kappa}}_{i}(\tau)-Q_{i} \check{\breve{A}}_{\perp}\left(\tau, \vec{\eta}_{i}(\tau)\right)\right)^{2}}-\right. \\
& \left.+i \frac{Q_{i} \vec{\xi}_{i}(\tau) \times \vec{\xi}_{i}(\tau) \cdot \check{\vec{B}}\left(\tau, \vec{\eta}_{i}(\tau)\right)}{2 \sqrt{m_{i}^{2}+\breve{\breve{\kappa}}_{i}^{2}(\tau)}}-i \frac{Q_{i} \check{\vec{\kappa}}_{i}(\tau) \cdot \vec{\xi}_{i}(\tau) \vec{\xi}_{i}(\tau) \cdot \check{\vec{E}}_{\perp}\left(\tau, \vec{\eta}_{i}(\tau)\right)}{\left(m_{i}+\sqrt{\left.m_{i}^{2}+\breve{\breve{\kappa}}_{i}^{2}(\tau)\right)} \sqrt{m_{i}^{2}+\breve{\vec{\kappa}}_{i}^{2}(\tau)}\right.}\right]+ \\
& +\sum_{i \neq j}\left[\frac{Q_{i} Q_{j}}{4 \pi\left|\vec{\eta}_{i}(\tau)-\vec{\eta}_{j}(\tau)\right|}-\right. \\
& \left.-i \frac{Q_{i} Q_{j} \check{\vec{\kappa}}_{i}(\tau) \cdot \vec{\xi}_{i}(\tau) \vec{\xi}_{i}(\tau) \cdot\left(\vec{\eta}_{i}(\tau)-\vec{\eta}_{j}(\tau)\right)}{4 \pi\left|\vec{\eta}_{i}(\tau)-\vec{\eta}_{j}(\tau)\right|^{3}\left(m_{i}+\sqrt{m_{i}^{2}+\check{\vec{\kappa}}_{i}^{2}(\tau)}\right) \sqrt{m_{i}^{2}+\check{\breve{\kappa}}_{i}^{2}(\tau)}}\right]+ \\
& +\int d^{3} \sigma \frac{1}{2}\left[\check{\vec{E}}_{\perp}^{2}+\check{\overrightarrow{\vec{B}}}^{2}\right](\tau, \vec{\sigma}) \text {. }
\end{aligned}
$$

We then studied the coupled Hamilton equations for the particles and the electro-magnetic field. By integrating the equations for the electro-magnetic field, we can express the trans- 
verse vector potential as the sum of a pure radiation term plus the transverse LienardWiechert term [29], which depends on the particles and on the choice of the Green function (retarded, advanced, symmetric,..). Due to the semi-classical approximation $Q_{i}^{2}=0$, each particle creates a transverse Grassmann-valued vector potential, so that a single particle cannot irradiate energy (it is a quantity quadratic in the fields) since it is of order $Q_{i}^{2}=0$. This solves at the semi-classical level the causality problems of the Abraham-Lorentz-Dirac equation. However, if we have various particles and we do not use their equations of motion (namely we consider them as sources), then there is emission of energy (it is of order $Q_{i} Q_{j}$, coming from interference terms) and we recover the Larmor formula in the wave zone. This is enough to explain the asymptotic radiation from a region containing macroscopic sources.

Independently from that, we can consider the Lienard-Wiechert solutions at some point P of space-time: they depend on the delayed (retarded, advanced, symmetric,..) times of the particles creating them. We can develop these delayed times so to re-express the LienardWiechert solutions only in terms of quantities evaluated on the Wigner hyperplane through the point P. A priori we get expressions which depend on the transverse potential at that time and on its time derivatives of any order (ordinary and higher accelerations) evaluated at that time (like it happens in Feynman-Wheeler electrodynamics if one make an equal time development of its integro-differential equations of motion). However, by using the equations of motion of the particles, we can show that only the transverse vector potential and its first time derivative (the velocity) survive: all the accelerations are multiplied by $Q_{i}^{2}=0$. Moreover, we get the same result whichever is the Green function considered (retarded, advanced, symmetric,...). It is now possible to express the semi-classical LienardWiechert transverse vector potential and the electric and magnetic fields in terms of the positions and momenta of the particles. Therefore, Grassmann-valued electric charges allow to extract the semi-classical action-at-a-distance potential hidden in the electro-magnetic interaction notwithstanding it is an interaction with delay, so that no anticipation survives at the semi-classical level.

Then we make a canonical reduction to the sector of bound states, in which only particle degrees of freedom are present. This is done by forcing the electro-magnetic fields in the Hamiltonian to coincide with the semi-classical Lienard-Wiechert solution. After having found the new canonical variables for the particles in this reduced phase space, we can re-express the Hamiltonian in terms of them. The final reduced Hamiltonian contains: a) 
the relativistic kinetic energy of the positive energy particles; b) the regularized Coulomb potential; c) the semi-classical Darwin potential with all its relativistic corrections. While in the spinless case the lowest order in $1 / c^{2}$ of the semi-classical Darwin potential coincides with the standard Darwin potential [30], in the spinning case at the same order we get an expression for such potential, which in the 2-body case with arbitrary masses after quantization coincides with the potential which was obtained from Bethe and Salpeter 31] starting from quantum field theory in an instantaneous approximation. All the spin-orbit and spin-spin terms for positronium $\left(m_{1}=m_{2}\right)$, muonium $\left(m_{1} \neq m_{2}\right)$ and hydrogen-like atoms $\left(m_{1} \rightarrow \infty\right)$ are reproduced.

\section{CONCLUSION}

Therefore we have discovered a way to find the semi-classical potential for a Cauchy problem which corresponds to the static and non-static contributions coming from the Feynman diagrams with an one-photon exchange. Let us remark that Feynman diagrams correspond to a Dirichlet problem which has anticipation: we are able to re-formulate it as a Cauchy problem without anticipation at the semi-classical level. Anticipation is pushed to the radiative corrections and many-photon exchanges, which take into account the delay.

We are now planning to redo the calculations on arbitrary space-like hyper-sufaces to study how accelerated observers describe the radiation emitted by particles. We will also try to extend the method to the quark model (strong interaction bound states of quarks with Grassmann-valued color charges [32]) to see whether it is possible to demonstrate the confinement of quarks with these semi-classical methods. The analogous problem in linearized tetrad gravity [22] with a perfect fluid as matter will push us to find the physical Hamiltonian of our gauge containing the relativistic action-at-a-distance potentials and the tidal interactions, and to determine the relativistic quadrupole emission formula. This would clarify the status of anticipatory effects in gravitational physics.

In conclusion we begin to understand some unexpected features of the semi-classical approximation in the rest-frame instant form like the elimination of anticipation. The role played by the Grassmann quantities is intriguing, because we do not understand the quantization of charges and which is the connection of their regularizing properties with the standard procedure of regularization in quantum field theory. Maybe the presence of a physical 
cutoff (the Møller radius) will help to clarify the last point. Is it possible to go beyond the semi-classical approximation and to replace the anticipatory many-photon exchanges and the radiative corrections with higher order potentials for a Cauchy problem without anticipation? Is a consistent addition of gravity going to change things qualitatively? Does the gravitational mass (the charge of gravitation) arise through some quantization and symmetry breaking mechanisms as it is suggested by special relativistic particle theory or has a completely different origin and a completely different semi-classical behaviour? No answer to these questions is known at present.

[1] G.Longhi and L.Lusanna, Phys.Rev. D34, 3707 (1986). G.Longhi, L.Lusanna and J.M.Pons, J.Math.Phys. 30, 1893 (1989).

[2] L.Lusanna, "Towards a Unified Description of the Four Interactions in Terms of DiracBergmann Observables", invited contribution to the book "Quantum Field Theory: a 20th Century Profile", of the Indian National Science Academy, ed.A.N.Mitra, foreward F.J.Dyson (Hindustan Book Agency, New Delhi, 2000) (hep-th/9907081).

[3] J.Stachel, The Meaning of General Covariance, in Philosophical Problems of the Internal and External Worlds, Essays in the Philosophy of A.Grünbaum, eds. J.Earman, A.I.Janis, G.J.Massey and N.Rescher (Pittsburgh Univ. Press, Pittsburgh, 1993). How Einstein Discovered General Relativity: a Historical Tale with Some Contemporary Morals, in Proc. GR11, ed. M.A.H.MacCallum (Cambridge Univ.Press, Cambridge, 1987).

[4] C.Rovelli, Class.Quanyum Grav. 8, 297 and 317 (1991).

[5] L.Lusanna and M.Pauri, General Covariance and the Objectivity of Space-Time PointEvents: The Role of Gravitational and Gauge Degrees of Freedom in General Relativity (gr-qc/0301040). M.Pauri and M.Vallisneri, Ephemeral Point-Events: is there a Last Remnant of Physical Objectivity?, essay for the 70th birthday of R.Torretti, 2002(gr-qc/0203014). L.Lusanna, Space-Time, General Covariance, Dirac-Bergmann Observables and Non-Inertial Frames, talk at the 25th Johns Hopkins Workshop 2001: A Relativistic Space-Time Odyssey (gr-qc/0205039).

[6] L.Lusanna, The Rest-Frame Instant Form of Metric Gravity, Gen.Rel. and Grav. 33, 1579 (2001) (gr-qc/0101048). 
[7] L.Lusanna and S.Russo, A New Parametrization for Tetrad Gravity, Gen.Rel.Grav. 34, 189 (2002) (gr-qc/0102074).

[8] R.De Pietri, L.Lusanna, L.Martucci and S.Russo, Dirac's Observables for the Rest-Frame Instant Form of Tetrad Gravity in a Completely Fixed 3-Orthogonal Gauge, Gen.Rel.Grav. 34, 877 (2002) (gr-qc/0105084).

[9] D.Christodoulou and S.Klainerman, The Global Nonlinear Stability of the Minkowski Space (Princeton, Princeton, 1993).

[10] L.Lusanna, Phys.Rep. 185, 1 (1990); Riv. Nuovo Cimento 14, n.3 (1991), 1; J.Math.Phys. 31, 2126 (1990); J.Math.Phys. 31, 428 (1990). Comtemp.Math. 132, 531 (1992).

[11] P.A.M.Dirac, Can.J.Math. 2, 129 (1950); Lectures on Quantum Mechanics, Belfer Graduate School of Science, Monographs Series (Yeshiva University, New York, N.Y., 1964).

J.L.Anderson and P.G.Bergmann, Phys.Rev. 83, 1018 (1951). P.G.Bergmann and J.Goldberg, Phys.Rev. 98, 531 (1955).

M.Henneaux and C.Teitelboim, Quantization of Gauge Systems (Princeton University Press, Princeton, 1992).

[12] S.Shanmugadhasan, J.Math.Phys. 14, 677 (1973). L.Lusanna, Int.J.Mod.Phys. A8, 4193 (1993).

[13] J.Stewart, Advanced General Relativity (Cambridge Univ. Press, Cambridge, 1993).

[14] D.M.Dubois, Review of Incursive, Hyperincursive and Anticipatory Systema - Foundation of Anticipation in Electromagnetism, in CASYS'99, Third Int.Conference, ed.D.M.Dubois (AIP Conference Proceedings 517).

[15] C.Itzykson and J.B.Zuber, Quantum Field Theory (McGraw-Hill, New York, 1985).

[16] R.P.Feynman and J.A.Wheeler, Rev.Mod.Phys. 17, 157 (1945); 21, 425 (1949).

[17] C.Callender and N.Huggett (Eds.), Physics meets philosophy at the Planck scale (Cambridge Univ.Press, Cambridge, 2001).

[18] L.Lusanna, The $N$ - and 1-Time Classical Descriptions of N-Body Relativistic Kinematics and the Electromagnetic Interaction, Int.J.Mod.Phys. A12, 645 (1997).

[19] D.Alba, L.Lusanna and M.Pauri, Center of Mass and Rotational Kinematics for the Relativistic N-Body Problems in the Rest-Frame Instant Form, J.Math.Phys. 43, 1677 (2002) (hep-th/0102087), Dynamical Body Frames, Orientation-Shape Variables and Canonical Spin Bases for the Non-Relativistic N-Body Problem, J. Math. Phys. 43, 373 (2002) 
(hep-th/0011014) and Multipolar Expansions for the Relativistic N Body Problem in the RestFrame Instant Form, submitted to J.Math.Phys. (hep-th/0103092).

[20] P.A.M.Dirac, Rev.Mod.Phys. 21 (1949) 392.

[21] C.Møller, The Theory of Relativity (Oxford Univ.Press, Oxford, 1957).

[22] J.Agresti, R.DePietri, L.Lusanna and L.Martucci, Hamiltonian Linearization of the RestFrame Instant Form of Tetrad Gravity in a Completely Fixed 3-Orthogonal Gauge: a Radiation Gauge for Background-Independent Gravitational Waves in a Post-Minkowskian Einstein Space-Time, to appear in Gen.Rel.Grav. (gr-qc/0302084).

[23] H.Crater and L.Lusanna, The Rest-Frame Darwin Potential from the Lienard-Wiechert Solution in the Radiation Gauge, Ann.Phys.(NY) 289, 87 (2001) (hep-th/0001046).

[24] D.Alba, H.Crater and L.Lusanna, The Semi-Classical Darwin Potential for Spinning Particles in the Rest-Frame Instant Form: Two Body Bound States with Spin 1/2 Constituents, Int.J.Mod.Phys. A16, 3365 (2001) (hep-th/0103109).

[25] A.Barducci, R.Casalbuoni and L.Lusanna, Nucl.Phys. B124, 93 (1977).

[26] A.Barducci, R.Casalbuoni and L.Lusanna, Lett.Nuovo Cim. 19, 581 (1977).

[27] F.A.Berezin and M.S.Marinov, Ann.Phys.(NY) 104, 336 (1977). A.Barducci, R.Casalbuoni and L.Lusanna, Nucl.Phys. [FS2] B180, 141 (1981).

[28] F.Bigazzi and L.Lusanna, Int.J.Mod.Phys. A14, 1429 (1999) (hep-th/9807052).

[29] D.Alba and L.Lusanna, Int.J.Mod.Phys. A13, 2791 (1998).

[30] H.W.Crater and D.Yang, J.Math.Phys. 32, 2374 (1991). X. Jaen, J. Llosa, and A. Molina, Phys. Rev. D34, 2302 (1986). R. A. Moore and T. C. Scott, Phys. Rev. A46, 3637 (1992). V.N Golubenkov and A. Ia. Smorodinski, Sov. Phys. JETP 31, 330 (1956)

[31] H.A.Bethe and E.E.Salpeter, Quantum Mechanics of One and Two Electron Atoms (Academic Press, New York, 1957) p 193. T.P.Das, Relativistic Quantum Mechanics of Electrons (Harper and Row, New York, 1973). G.Breit, Phys. Rev. 34, 553 (1929)

[32] D.Alba and L.Lusanna, Int.J.Mod.Phys.A13, 3275 (1998) (hep-th/9705156). L.Lusanna, 1995 Int.J.Mod.Phys. A10, 3531 and 3675. 